\title{
Impact Force Identification By Using Modal Transformation Method For Automobile Test Rig
}

\author{
Abdul Ghaffar Abdul Rahman ${ }^{1, a}$, Khoo Shin Yee ${ }^{2, b}$, Zubaidah Ismail $^{3, c}$, \\ Chong Wen Tong ${ }^{2, \mathrm{~d}}$ and Siamak Noroozi ${ }^{4, e}$
}

\author{
${ }^{1}$ Faculty of Mechanical Engineering, University of Malaysia Pahang, 26600 Pekan, Pahang, \\ Malaysia \\ ${ }^{2}$ Department of Mechanical Engineering, Engineering Faculty, University of Malaya, 50603 Kuala \\ Lumpur, Malaysia \\ ${ }^{3}$ Department of Civil Engineering, Engineering Faculty, University of Malaya, 50603 Kuala Lumpur, \\ Malaysia \\ ${ }^{4}$ School of Design, Engineering \& Computing, Bournemouth University, Poole, Dorset, BH12 5BB, \\ UK \\ adragrahman@yahoo.com, ${ }^{b}$ mikeson.khoo@yahoo.com; khooshinyee@um.edu.my, \\ czu_ismail@um.edu.my, ${ }^{d}$ chong_wentong@um.edu.my, ${ }^{\text {es }}$ snoroozi@bournemouth.ac.uk
}

Keywords: correlation; frequency response function; impact force identification; lightweight structure; modal transformation method; operating deflection shape

\begin{abstract}
In the automobile industry, impact force is the main cause for material fatigue in lightweight vehicles. Bump-excited impact force is the most common case, which causes damage to vehicles and reduces the quality of the ride. Force identification is important to reflect the structure's health so that action such as structure modification can be taken before material fatigue. However, direct measurement by using force transducer is not practical due to difficulty in force sensor configuration. A methodology utilizing Operating Deflection Shape (ODS) analysis, Frequency Response Function (FRF) measurement and Modal Transformation Method (MTM) to evaluate the dynamic force is proposed here. This method is called indirect force measurement by using inverse technique. The performance of this approach was demonstrated via experiment. From the measured responses and measured dynamic characteristics of an automobile test rig, a real time mathematical manipulation can generate the system's input force. The force location is known in priori for impact excitation and therefore the inverse problem is well-posed. This method was tested using different force location with unique input force. It shows that high quality of curve fitting to extract the modal parameters such as damped natural frequency, modal damping and residue mode shape is essential to obtain a high accuracy force determination result.
\end{abstract}

\section{Introduction}

Impact force is the main cause for material fatigue in lightweight structure especially vehicles in the automobile industry [1]. Bump-excited impact force is the most common case, which causes damage to vehicles and reduces the quality of the ride in frequency range of 10 to $100 \mathrm{~Hz}$ [2]. In time domain, impact force is a high magnitude force that excites on a structure for a short duration. Considering the dynamic effects in frequency domain, it causes broadband excitation and excites several vibration modes of a structure simultaneously. The load-carrying capability of a structure will be degraded if this type of force is exciting a structure for a sufficient period depending on the magnitude of force. Therefore impact force identification is important to reflect the structure's health so that action such as structure modification can be taken before material fatigue. However, 
direct measurement by using force transducer is not practical due to difficulty in force sensor configuration. Besides that, installation of a force sensor at the structure's junction, link or support is not feasible. This is because it could influence the boundary condition of a structure and hence change the dynamic characteristics [3].

On the other hand, it would be convenient to measure the response of a structure instead of measuring the unknown impact force directly. By measuring response and dynamic characteristics or frequency response function (FRF) of a structure using operating deflection shape (ODS) analysis and FRF measurement, analysis involved backtracking to determine the force causing the problem can be done. It is known that this inverse identification problem is ill-conditioned in most practical cases because not all the initial conditions are known [4]. Besides that, the ill-conditioned problem is caused by system response which is defined only at a few discrete points [5]. In general system response is a continuous function of spatial coordinates. An inverse problem is ill conditioning if small perturbations in measured response can cause large changes in calculated force. Measurement errors are amplified during the inversion operation and produce meaningless force identification result.

A lot of methods have been developed to solve the ill-conditioned problem in frequency domain. In this study, modal coordinate transformation approach or modal transformation method (MTM) is used because it can reduce the computing time in the inversion operation [6]. MTM is applied to synthesize FRF from modal parameters extracted from single column raw FRF matrix by using general curve fitting algorithm. In this paper, impact force determination by using MTM is demonstrated via experimental verification. The effectiveness of MTM in impact force determination with and without the knowledge of impact location is examined. The robustness of this force identification method is tested using different force location with unique input force. Besides that, the curve fitting result is tested by using correlation tool to show its impact on force determination result.

\section{Materials and Methods}

Set-up of experiment equipment. A rectangular shape test rig with four ground supports was simulated as vehicle's body and referred to as automobile test rig. Fifteen accelerometers were attached on the rig and numbering as shown in Fig. 1. The rig was used to acquire the responses from 15 discrete locations simultaneously. A modally tuned impact hammer was used to record the time history of impact excitation. These input and output signals were connected to a laptop through a data acquisition (DAQ) system. Hence, post-processing of the data was done by using DASYLab ${ }^{\circledR}$ and MATLAB ${ }^{\circledR}$ software.

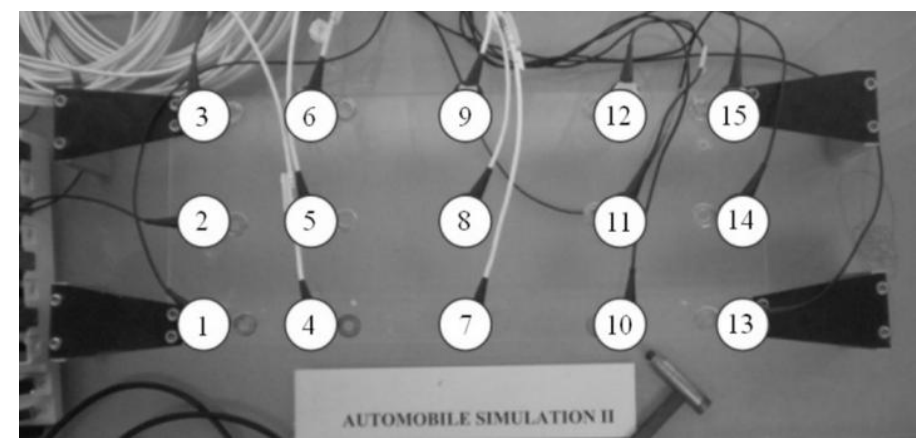

Fig. 1. Point numbering on the automobile test rig. 
Impact Force Determination By Using Modal Transformation Method. For forced vibrations of $n$ DOF system with viscous damping, the general solution of the linear time invariant vibration system is expressed as follows:

$$
\{\underset{n \times 1}{\ddot{\boldsymbol{X}}(\omega)}\}=\underset{n \times n}{[\mathbf{H}(\omega)]} \underset{n \times 1}{\boldsymbol{Q}(\omega)}\} .
$$

where $[\mathbf{H}(\omega)]$ is an $n$ by $n$ square FRF matrix. $\{\ddot{\boldsymbol{X}}(\omega)\}$ and $\{\boldsymbol{Q}(\omega)\}$ are $n$ by 1 acceleration and force vectors. $\omega$ is angular frequency with unit rads $^{-1}$.

Single column ( $n$ by 1 ) raw FRF matrix is sufficient to extract the modal parameters such as damping ratio, residue mode shape and damped natural frequency by using curve fitting algorithm. Given number of response, mode and force measurements are $n, m$ and $f z$ respectively, MTM is applied to synthesize the FRF as shown in Eq. 2.

$$
\left.\underset{n x f z}{[\mathbf{G}(\omega)]}=\underset{n \times m}{\left[\mathbf{\Phi}_{\mathbf{N}}\right]}\left[\because 1 /\left(-\omega^{2}+\underset{m \times m}{2 i \omega \zeta_{k}} \omega_{0 k}+\omega_{0 k}^{2}\right) \cdot\right] \underset{m x f z}{\left[\mathbf{\Phi}_{\mathbf{N}}\right.}\right]^{\mathrm{T}}
$$

where $[\mathbf{G}(\omega)]$ is an $n$ by $f z$ synthesized FRF matrix. $\left[\boldsymbol{\Phi}_{\mathbf{N}}\right]$ is $n$ by $m$ unit modal mass (UMM) mode shape matrix due to response DOF. It can be obtained from residue mode shape as follows reference [7]. $\left[\boldsymbol{\Phi}_{\mathbf{N}}\right]^{\mathrm{T}}$ is $m$ by $f z$ UMM mode shape matrix due to force DOF and it is transpose of $\left[\boldsymbol{\Phi}_{\mathbf{N}}\right] . \omega_{0 k}$ is the $k^{\text {th }}$ mode natural frequency where $k=1,2, \ldots, m . \zeta_{k}$ is the $k^{\text {th }}$ mode damping ratio. [ $\because \ddots$ ] is a diagonal matrix.

Unknown force can be recovered by multiplying pseudo-inverse, pinv of synthesized FRF matrix to measure response vector using Eq. 3. To obtain a least square solution of force determination, it must satisfy $n \geq m \geq f z$.

$$
\{\underset{f z \times 1}{\boldsymbol{Q}(\omega)}\}=\operatorname{pinv}\{[\underset{n \times f z}{\mathbf{G}(\omega)]\}} \underset{n \times 1}{\ddot{\boldsymbol{X}}(\omega)}\}
$$

In this study, the force identification method was tested in two cases: one with and the other without the knowledge of impact location. In both cases, a single unknown impact force acting at point 1 on the test rig was estimated from 15 acceleration sensors. This procedure was repeated for different locations such as points 3,13,15. Correlation between imaginary part of mean of synthesized and raw FRFs for force DOF 1, 3, 13 and 15 were calculated to study the effectiveness of curve fitting on force determination results.

\section{Results and Discussions}

For the case where impact location was unknown, the force was estimated at 15 locations. The calculated force due to impact at point 1 is shown in Fig. 2 and other forces due to impact at points 3, 13 and 15 were not shown because the results were similar. It was found that the magnitudes, functions and locations of estimated forces were incorrect. In fact, actual force excited at one of the 15 locations, left forces at other locations to be zero.

In case 2, four sets of different calculated forces acting at four different locations were compared with its actual forces as shown in Fig. 3. It was found that the force determination problem had become well-posed once impact location was known in a priori. The impact function of recovery force was correct but the accuracy of magnitude was not satisfied. Fig. 3 shows that the time history of estimated force at point 15 was the closest to the measured force. 


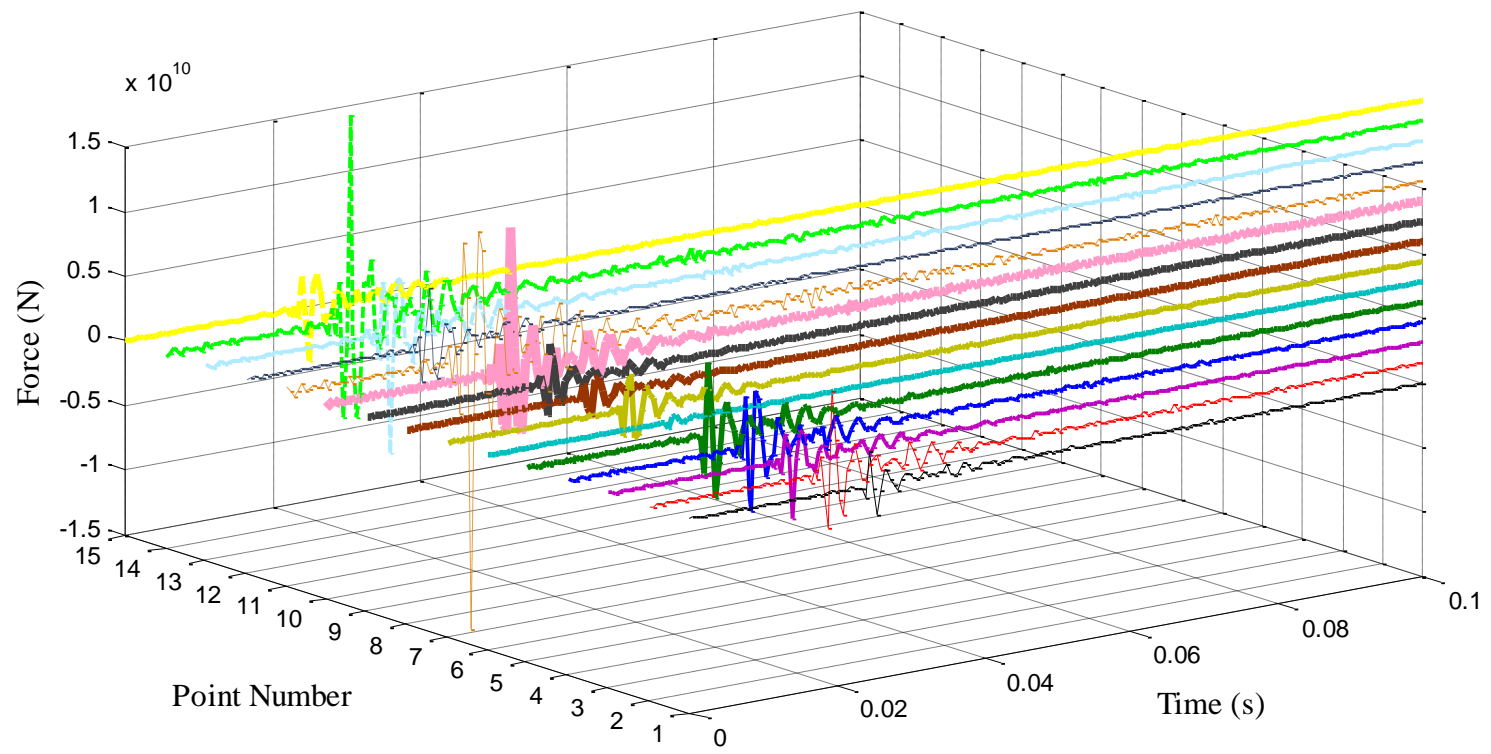

Fig. 2. Calculated force history and impact location for original impact force excite at point 1 on automobile test rig for 15 discrete locations.
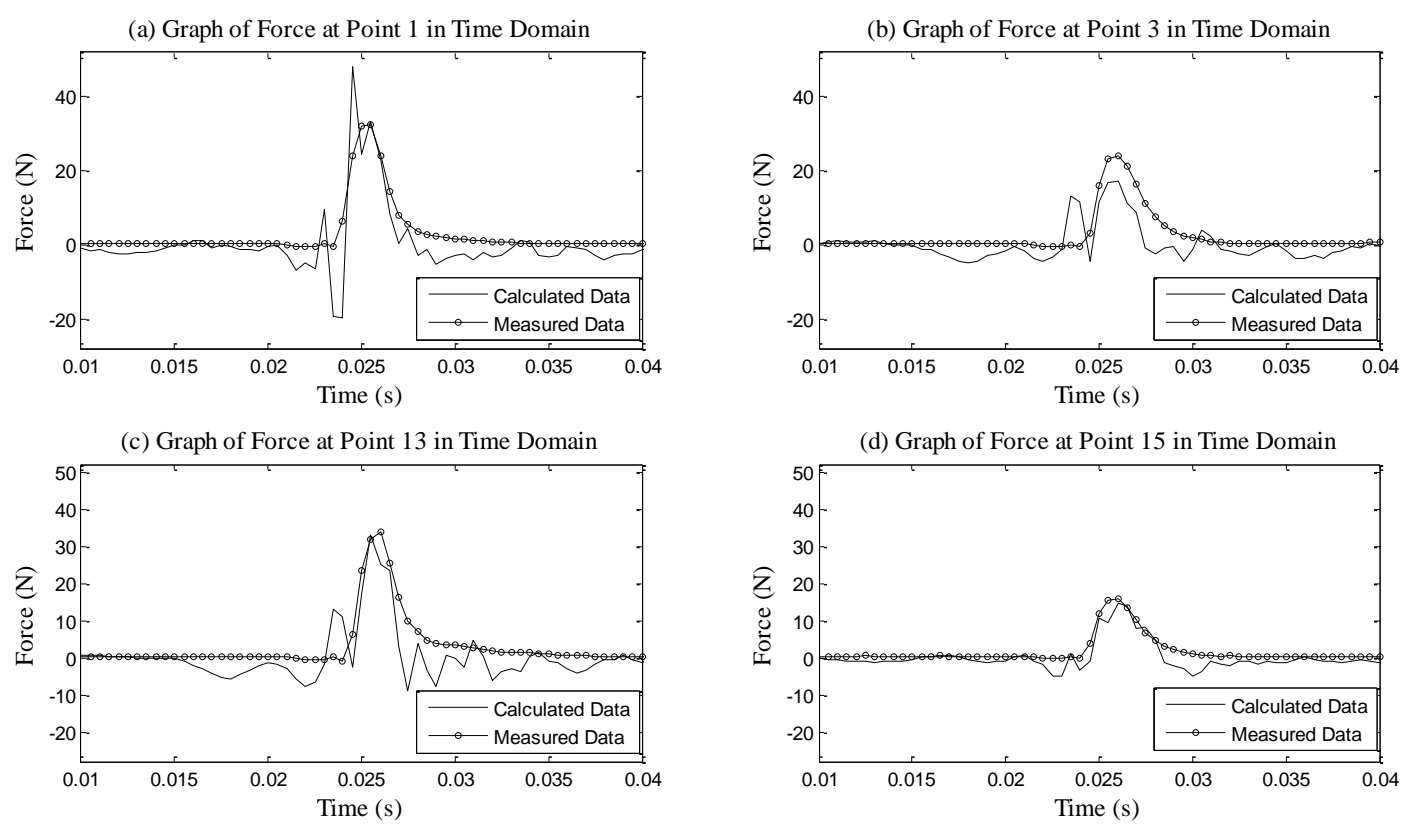

Fig. 3. Comparison between four sets of different calculated forces acting at four different locations with the measured force: (a) force at point 1 (b) force at point 3 (c) force at point 13 (d) force at point 15.

Correlation between imaginary part of mean of synthesized and raw FRFs for force DOF 1, 3, 13 and 15 for a half-band spectrum is shown in Fig. 4. Correlation in range 0.9 -1.0 indicates the curve fitting result is excellent to synthesize the FRFs. In fact, curve fitting result is satisfied if the imaginary parts of the synthesized FRFs match the "shape" of measured FRFs [8]. It shows that result of force DOF 15 had the best correlation followed by 1, 3 and 13. Note that the correlation drops by increasing the frequency due to contamination of measurement noise and incorrect contribution of modes. Combining results from Figs. 3 and 4, shows that curve fitting result influences the accuracy of the reconstructed force. Therefore care and attention on curve fitting algorithm must be taken to enhance the accuracy of force determination result. 


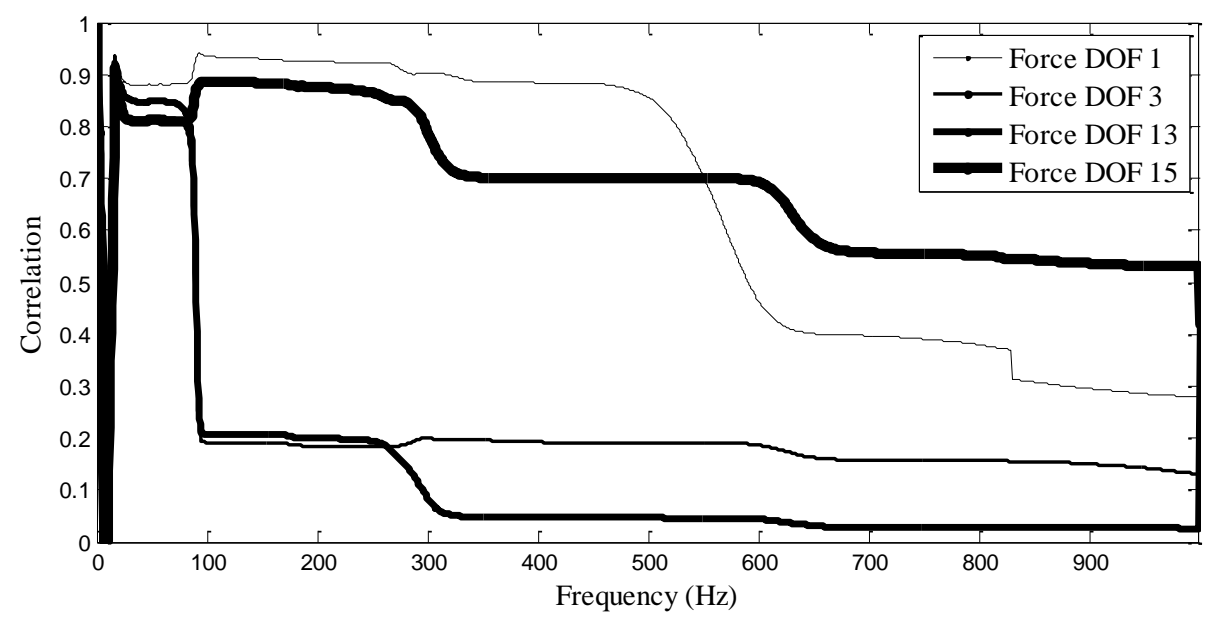

Fig. 4. Graph of correlation between imaginary part of mean of synthesized and measured FRFs at different force DOFs versus frequency.

\section{Summary}

In this study, impact force identification by using modal transformation method has been examined in two cases: one with and the other without impact location information. Impact force determination problem becomes well-conditioned if impact location is known. Higher correlation of curve fitting result must be obtained to improve the impact force determination result.

\section{References}

[1] D.M. Lilley, A. Kapoor, G. Gu, Calculation of dynamic impact loads for railway bridges using a direct integration method, Proceedings of the Institution of Mechanical Engineers, Part F: Journal of Rail and Rapid Transit. 222 (2008) 385-398.

[2] T. Lee, B. Kim, Vibration analysis of automobile tire due to bump impact, Applied Acoustics. 69 (2008) 473-478.

[3] J.-Y. Yoon, and R. Singh, Estimation of interfacial forces in a multi-degree of freedom isolation system using a dynamic load sensing mount and quasi-linear models, Journal of Sound and Vibration. 330 (2011) 4429-4446.

[4] Z. Boukria, P. Perrotin, A. Bennani, Experimental impact force location and identification using inverse problems: application for a circular plate, International Journal of Mechanics. 5 (2011) 48-55.

[5] R.J. Hundhausen, D.E. Adams, M. Derriso, P. Kukuchek, R. Alloway, Transient loads identification for a standoff metallic thermal protection system panel, in: 23rd International Modal Analysis Conference (IMAC XXIII), Orlando, Florida (2005).

[6] B. Wang, Prediction of impact and harmonic forces acting on arbitrary structures: theoretical formulation, Mechanical Systems and Signal Processing. 16 (2002) 935-953.

[7] M. Richardson, C. Jamestown, Modal mass, stiffness and damping, Vibrant Technology. Inc., Jamestown, CA, (2000) 1-5.

[8] Vibrant Technology,Inc., Multiple reference curve fitting to find closely coupled modes, App. Note \# 15, April,2001. 